Products and Services In Practice is provided to readers using text and images from the manufacturer, supplier or distributor and does not imply endorsement by $B D J$ In Practice. Normal and prudent research should be exercised before purchase or use of any product mentioned.

Please send product and services news through to David Westgarth, $B D J$ In Practice via: David.Westgarth@bda.org

\section{A practice differentiator}

Straumann coDiagnostiX is more than just implant planning software. It's a practice differentiator.

The state-of-the-art software offers numerous measurement and planning functionalities, while automatically detecting nerve canals for added peace of mind. It also enhances patient communication by providing enhanced visualisation for improved understanding and treatment acceptance. In addition, it directly integrates with the Straumann P10 - a capsule-based 3D printer designed to fabricate surgical guides in-practice.

The Straumann coDiagnostiX Easy offers a simplified software interface for the complete workflow, which is ideal for clinicians looking to start their journey into guided surgery.

With more than 20 years development behind it, the Straumann coDiagnostiX offers superior treatment planning for minimal risk. For sophisticated but user-friendly diagnostics and supported implant planning that will help you stand out from the crowd, make coDiagnostiX your software of choice.

For more information visit at www. straumann-uk.co/cares-chairside.

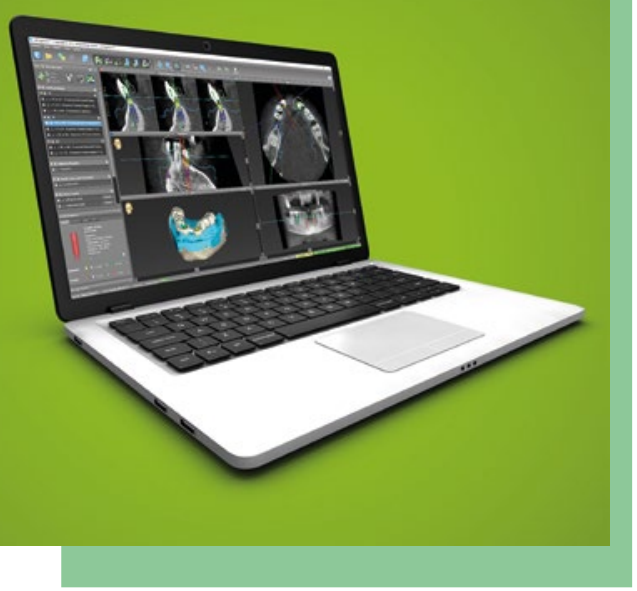

\title{
The right environment to thrive
}

If you're looking for the right environment in which you could truly thrive, consider joining the Colosseum Dental family. They have newlyrefurbished, modern dental clinics across the UK and offer every opportunity for job security and career progression.

In fact, support, training and education are at the heart of what they stand for. They provide access to an array of CPD topics and additional training courses for all associates. They also provide any further support you and your practice colleagues need to deliver exceptional patient care.

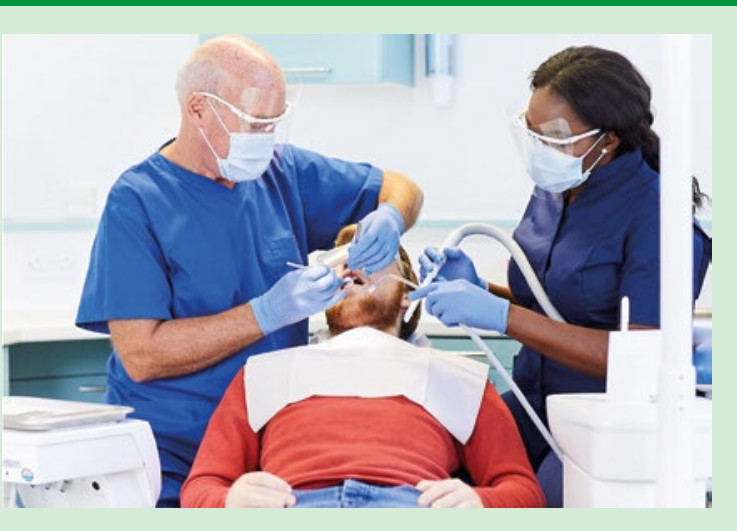

To see if Colosseum Dental could provide the right environment for you to excel, please send your CV to resourcing@ colosseumdental.co.uk.

\section{Empowering dentists to enhance patient care}

If you're looking for a fresh challenge by moving into a new area of dentistry, why not consider training in dental implantology with Ucer Education?

Led by Specialist Oral Surgeon, Professor Cemal Ucer, Ucer Education provides various learning opportunities for modern clinicians. These include the PG Cert in Implant Dentistry, designed to provide a comprehensive, multi-system introduction to the topic with both theoretical and practical guidance.

The 12-month course is presented by

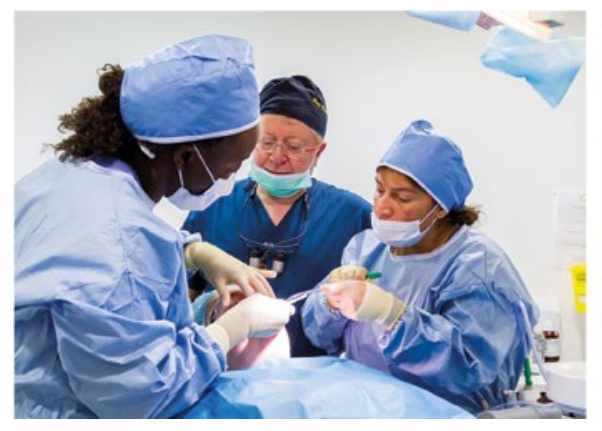

a first-class teaching faculty alongisde the cutting-edge facilities of the ICE Postgraduate Hospital in Manchester. It also concludes in a formal Level 7 qualification awarded by EduQual.

Get empowered to enhance your patient care with Ucer Education.

For more information on the PG Cert in Implant Dentistry from Ucer Education supported by Geistlich, Megagen, Neoss, TRI Implants and General Medical - visit www.ucer.education or contact Prof Ucer at ice@ucer.uk.

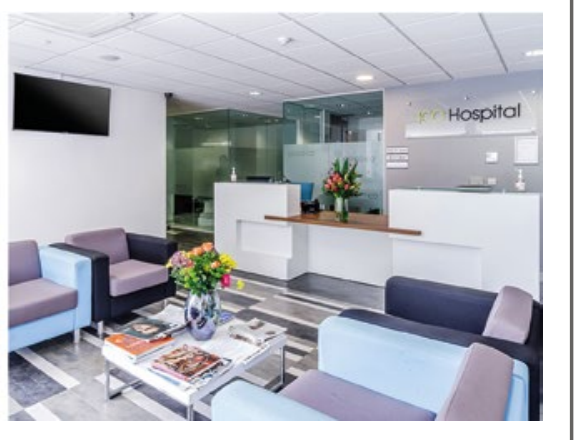

